\section{Die Bedeutung der Kategorie Wissen für den Wandel der Arbeit}

\author{
von Hermann Kocyba, Institut für \\ Sozialforschung
}

Auch wenn Wissen heute als entscheidender Wettbewerbsfaktor gilt, löst sich Produktionsarbeit nicht einfach in Wissensprozesse auf. Wissen erweist sich nur in Kombination mit Kapital und Arbeit als wertschöpfend, es tritt nicht an deren Stelle. Neue partizipative Steuerungskonzepte verändern gleichzeitig die traditionelle Wissensarchitektur des Unternehmens, tendenziell wird jeder zum Wissensarbeiter. Die zunehmende Wissensabhängigkeit wirtschaftlicher Prozesse geht nicht nur einher mit der Gefahr, zu wenig, zu spät oder das Falsche zu wissen, sondern schafft neue Risiken in Gestalt von Entscheidungsblockaden durch übergroße Datenmengen. Dies erfordert Selektionsfilter. Vor diesem Hintergrund erzeugt die neue Wissenspolitik im Unternehmen eine Tendenz zu Visualisierung, Simplifizierung und Standardisierung und gefährdet damit erreichte Partizipationsgewinne.

\section{Einführung}

Dass Wissen zur entscheidenden Produktivkraft moderner Ökonomien geworden ist und dem Einsatz der Wissensressourcen eines Unternehmens entscheidende Bedeutung für dessen Wettbewerbsfähigkeit zukommt, erscheint uns mittlerweile trivial. Seit Jahren sind Bestseller auf dem Markt, die uns dabei helfen wollen, Wissen als brachliegende Unternehmensressource nutzbar zu machen (Nonaka, Takeuchi 1997) bzw. das Wissenskapital des Unternehmens als immateriellen Vermögenswert aufzuspüren, zu messen und zu steigern (Sveiby 1998). Managementexperten und zeitdiagnostisch ambitionierte Sozialwissenschaftler verkünden seit mehr als einem Jahrzehnt den Übergang von der Industrie- bzw. der ,Arbeitsgesellschaft" zu einem neuen Typus von „Wissensgesellschaft”.

Mit dem publizistischen Amtsantritt der „Wissensgesellschaft” allerdings lösen sich industrielle Produktion und Produktionsarbeit nicht einfach in Luft beziehungsweise in infor- mationstechnologisch vermittelte Kommunikation auf (Kocyba 1999). Das Thema „Arbeit in der Wissensgesellschaft" reduziert sich nicht auf die Frage der Ausbreitung eines bestimmten Typus von „Wissensarbeitern" und „Symbolanalytikern". Es geht nicht nur um die wachsende Bedeutung von Analysten, Wirtschaftsprüfern, Unternehmensberatern, Ingenieuren, Projektentwicklern, Wirtschaftsanwälten, Finanzdienstleistern oder Versicherungsmaklern. Wir beobachten gleichzeitig eine Aufwertung der Wissenskomponenten auch bei eher konventionellen Typen von Arbeit und eine Verschiebung des Verhältnisses von Planung, Steuerung und Kontrolle betrieblicher Prozesse einerseits und der operativen Umsetzung entsprechender Vorgaben innerhalb betrieblich organisierter Arbeitsprozesse andererseits. Auch in den Paradebranchen des tayloristischen Arbeitsregimes zeichnet sich eine neue, flexiblere Verbindung der Sphären von „Handarbeit” und „Kopfarbeit” ab (Kocyba, Vormbusch 2000).

Der Bedeutungszuwachs wissensbasierter Arbeit impliziert allerdings - anders als dies im Überschwang des Wissensgesellschaftsdiskurses gelegentlich formuliert wurde - nicht, dass Wissen unmittelbar an die Stelle von Arbeit oder von Kapital treten würde. Auch Wissensarbeit ist Arbeit. Der Wissensarbeiter wird nicht in abstracto für sein Wissen, sondern für dessen Nutzung innerhalb bestimmter, durch das Unternehmen definierter Einsatzkontexte bezahlt. Entgegen der in der Beraterliteratur propagierten These, für ökonomische Wertschöpfungsprozesse sei Wissen inzwischen wichtiger als die Faktoren Kapital und Arbeit (etwa im Anschluss an Drucker 1993), gilt es darauf zu insistieren, dass Wissen als solches, ohne mit Kapital und Arbeit kombiniert zu sein, keineswegs ,wertschöpfend" ist. Im Vergleich zu Arbeit und Kapital, wie sie auf anonymen Jedermannsmärkten verfügbar sind, werfen wissensspezifisch ,,veredelte" Formen von Arbeit oder Kapital höhere Renditen $\mathrm{ab}$ - aber es ist nicht unbedingt das „Jedermannswissen”, das den kritischen Wettbewerbsvorteil begründet, sondern wettbewerbsrelevantes, prozessspezifisches und in der Regel proprietäres Wissen. Wissen ist nicht gleich Wissen, wie die Wissensökonomie, die bislang häufig noch nicht einmal klar zwischen Wissen und Information zu unterschieden in der Lage ist, vielfach erst noch lernen muss. ${ }^{1}$ 


\section{Wissensabhängigkeit von Wertschöpfungsprozessen}

Trotz aller berechtigten Skepsis gegenüber der behaupteten Verdrängung des Wertschöpfungsfaktors Arbeit durch einen ganz neuen Wertschöpfungsfaktor namens Wissen ist eine Akzentverlagerung erkennbar, die einhergeht mit einer im Rahmen neuer Managementkonzepte erzeugten gesteigerten ,Sichtbarkeit” der Wissenskomponenten von Arbeit, die zunehmend auch die Dimension kommunikativer Aushandlungsprozesse einschließt (Minssen 1999; Vormbusch 2002). Menschliche Arbeitskraft wird zunehmend erst als praktische Verkörperung von Wissen ökonomisch interessant. Der Tendenz nach schwindet nicht nur die Bedeutung der ,Jedermannsarbeit”, sondern anscheinend auch die des ,Jedermannskapitals”; dieser Eindruck drängt sich zumindest auf, wenn man die teilweise die Milliardenschwelle überschreitenden Beträge betrachtet, mit denen sich die Manager erfolgreicher Hedge-Fonds ihr Wissen um die richtigen Anlageoptionen vergüten lassen). Die „,immateriellen Unternehmenswerte”, denen beispielsweise Sveiby nachspürt, verkörpern spezifische Verbindungen von Kapital und Expertise, die - ganz analog zum Fall personengebundener Qualifikationen als Verbindung von professioneller Expertise und subjektiver Arbeitsfähigkeit - nicht einfach unter Umgehung zeitintensiver Lernprozesse beliebig vervielfacht werden können (Sveiby 1998).

Auch diejenigen Beobachter, die der These eines durch die Entwicklung hin zur Wissensgesellschaft bewirkten radikalen Umbruchs der Arbeitswelt eher reserviert gegenüber stehen, bestreiten in aller Regel nicht, dass seit einigen Jahrzehnten ein Anstieg der Zahl hochqualifizierter, oftmals akademisch ausgebildeter Arbeitskräfte festzustellen ist. Die verstärkte Nachfrage nach qualifizierter Arbeit geht mit verstärkten Schwierigkeiten für gering qualifizierte Arbeit am Arbeitsmarkt einher. Strittig bleibt allerdings, in welchem Umfang hier wirklich ein unabweisbarer Bedarf an spezifischen Qualifikationen ausschlaggebend ist, wie sie in der Regel durch bestimmte Abschlüsse dokumentiert werden, oder, ob die Entwicklung nicht doch auf weite Strecken angebotsinduziert ist und insofern eher mit der Entwicklung des Bildungssystems als mit den Erfor- dernissen der Arbeitswelt zu erklären ist (Stehr 2003). Qualifikationen, die in modernen ITbasierten Bürotätigkeiten oder auch im Produktions-, Service- und Beratungsbereich erforderlich sind, sind nicht notwendig solche, die durch ein wissenschaftliches Studium erworben werden müssen. Häufig geht es eher um eine gewisse Artistik im Umgang mit Zeichen, Zahlen, Symbolen und Diagrammen, die als Bausteine oder Werkzeuge benutzt werden, ohne dass beispielsweise in einer Marketingpräsentation der Anspruch auf wissenschaftliche Überprüfbarkeit einzelner Aussagen erhoben würde.

Worin liegt - einmal abgesehen von ihrer nicht zu unterschätzenden Funktion für die Stabilisierung beruflicher Statushierarchien die praktische Bedeutung einer akademischen Ausbildung? In der Regel ja nicht darin, dass die betreffenden Mitarbeiter versuchen, organisatorische Prozesse im Unternehmen entsprechend der Logik kooperativer Wahrheitssuche umzugestalten. Auch dass sie in der Lage sind, sich zu Legitimationszwecken auf akademisches Wissen zu berufen, erklärt ihre Rolle nur teilweise. Gewiss ist es für ein Unternehmen von hohem Wert, wenn Mitarbeiter im Stande sind, zu erkennen, was an akademisch generiertem Wissen für ein bestimmtes Problem von Bedeutung sein könnte und wie es in einer konkreten Situation eingesetzt werden kann. Hierfür ist es möglicherweise vorteilhaft, wenn die betreffenden Mitarbeiter Erfahrung in Forschungskontexten haben, also Erfahrungen gesammelt haben im Umgang mit noch nicht „erkaltetem“, zur offizialisierten Lehrbuchform geronnenem Wissen. Aber leider ist dies nicht unbedingt das, was man an Hochschulen lernen kann - und zwar möglicherweise umso weniger, je stärker eine Ausrichtung auf reale oder vermeintliche Bedürfnisse der Wirtschaft das Curriculum bestimmt.

Vielfach allerdings ist für Unternehmen nicht akademisch generiertes Wissen entscheidend, sondern die Kompetenz, symbolgestützte Operationen in praktischen Kontexten einzusetzen. Marketing und Vertrieb erfordern ein spezifisches Wissen, das in der Regel gerade nicht akademischer Natur ist, das sich aber vor dem Hintergrund von Technologien als wichtig erweist, die auch für Wettbewerber verfügbar sind. Die Wissensabhängigkeit von Wertschöpfungsprozessen ist nicht unbedingt ein Indika- 
tor für die Bedeutung von im engeren Sinne wissenschaftlichem Wissen, entscheidend ist vielfach, wie bereits Hayek in seiner Analyse der gesellschaftlichen „Wissensteilung“ betonte, das praktische Wissen um die besonderen lokalen Umstände (Hayek 1976, S. 106 ff.).

Auch das Wissen, das für moderne Wertschöpfungsprozesse unverzichtbar ist, kann gleichzeitig angesichts immer leistungsfähigerer Informationstechnologien zum Problem und zum Ballast werden. Die Wissensabhängigkeit moderner Produktionsprozesse wird auch daran deutlich, dass Entscheidungsfähigkeit und Effizienz durch eine potenziell immer weiter anschwellende Informationsflut bedroht wird. Die Wahl der richtigen Kenngrößen erweist sich als entscheidendes Steuerungsmittel, um zu vermeiden, dass überquellende Datenmassen Entscheidungsprozesse sowohl des Managements als auch der dezentralen Einheiten blockieren. Mindestens ebenso wichtig wie die Frage des Wissenszugangs einerseits und informationeller Selbstbestimmung andererseits wird die Entwicklung geeigneter Selektionsfilter. „Wirklich knapp geworden sind die Kompetenzen, mit der Informationsflut etwas anzufangen." (Hack 1998, S. 719) „Notwendige Informationen nur bei Bedarf liefern”, forderte bereits der „Erfinder" des Toyota-Produktionssystems, das heißt des Prinzips der lean production (Ohno 1993, S. 75f). ,Schlanke Produktion“ impliziert nicht nur verringerte Fertigungstiefe, sondern mit der Abflachung betrieblicher Hierarchien gleichzeitig die Verlagerung von Verantwortung und Kompetenz hin zu den ausführenden Bereichen. Dies setzt die Möglichkeit voraus, auf Wissen zuzugreifen, das zuvor nur für das Management zugänglich war, und erfordert gleichzeitig die Verfügbarkeit wirksamer Filtermechanismen.

\section{Wissensprozesse und Arbeitsprozesse}

Die zunehmende Bedeutung der Wissensbasis von Arbeit führt nicht dazu, dass Arbeit sich inzwischen gänzlich im Hantieren mit Symbolen erschöpfte. Dies gilt auch für die wissenschaftliche Grundlagenforschung nicht, die durch einen komplexen Bezug auf Materialität und Stofflichkeit, auf Apparaturen und Instrumente charakterisiert ist und in der die Grenzziehung zwischen technischen und interpretativen Praktiken eher Ausdruck methodologischer
Normierungsbedürfnisse ist als eine in der Forschungspraxis greifbare Scheidelinie. Wissen und gegenständliches Tun bilden keinen Gegensatz. Lange vor allen modischen Diskursen über Wissensarbeit erforderten bereits vermeintlich simple handwerkliche Tätigkeiten spezifisches Wissen. Relativ neu gegenüber diesem traditionell-handwerklichen Modell der Verknüpfung von Arbeit und Wissen ist für die moderne „wissenschaftliche Betriebsführung“, dass dieses Wissen organisatorisch abgetrennt werden kann von den stofflich-energetischen Prozessen, die es abbildet, steuert und kontrolliert. Dieses Wissen, das alle Arbeitsprozesse begleitet, bezieht sich dabei nicht allein auf die technische Steuerung von Bearbeitungs- und Transportschritten, sondern auch auf

- die Abbildung als Kosten- und Wertprozess oder auf die Erfassung rechtlicher Dimensionen,

- die Wünsche und die Ansprachemöglichkeiten von Kunden,

- Möglichkeiten der Präsentation und der Verpackung,

- Kaufprozesse und dabei gewährte Bedingungen,

- Kenntnis der Produkte und der Vertriebsund Markenstrategien relevanter Mitbewerber.

Die Produktionsprozesse im engeren Sinne stellen nur einen Ausschnitt der insgesamt relevanten Wertschöpfungsaktivitäten dar. In dem Maße allerdings, in dem nicht mehr über Massenproduktion und deren Kostenvorteile allein Wettbewerbsvorteile zu erzielen sind und in dem gleichzeitig die organisatorische Trennung und Abschottung von Produktion, Marketing, Service usw. einer veränderten Organisation Platz macht, die beispielsweise die Kundenbeziehung als Innovationsquelle nutzen will, propagieren post-tayloristische Managementkonzepte die Reintegration von Arbeitsund Wissensprozess.

Dass etwas wissensbasiert ist, heißt in vielen Fällen, dass es auch anders oder besser organisiert werden könnte. Wissen ist der volatile Anteil an Prozessen, es kann schneller übertragen und modifiziert werden als im Normalfall die physischen Anlagen und Prozesskomponenten. Moderne Informationstechnologien machen es möglich, ohne zeitliche Verzö- 
gerung weltweit auf technische oder ökonomische Daten zuzugreifen - die Kunst ist freilich die, diese Informationen angemessen und zeitnah „rekontextualisieren“ zu können. ${ }^{2}$ In dem Maße, wie die Wettbewerbsfähigkeit von Unternehmen von der Fähigkeit abhängt, rasch und flexibel auf Veränderungen des Marktes, von Kostenstrukturen, Rohstoffen und verfügbaren technischen Möglichkeiten einzugehen, kommt der Reaktionsgeschwindigkeit besondere Bedeutung zu. Wer dagegen ausschließlich auf die traditionellen Vorteile standardisierter Massenproduktion setzen wollte, hätte schon vom Start weg verloren.

Soweit Wissen wettbewerbsrelevant ist, handelt es sich in der Regel nicht um allgemein zugängliches, dekontextualisiertes und standardisiertes explizites Wissen. Anders als die Rede von der Wissenschaft als „Wertschöpfungsfaktor" oder „Mehrwertquelle“ unterstellte, geht es nicht einfach darum, wissenschaftliches Wissen qua schlichter Übertragung anzuwenden. Um tatsächlich produktiv zu werden und um in einem spezifischen Wettbewerbsumfeld erfolgreich sein zu können, müssen unterschiedliche Wissenstypen gleichzeitig eingesetzt und auf eine sehr spezifische Weise miteinander und mit stofflich-physischen Prozessen und organisatorischen Praktiken gekoppelt werden. Dabei geht es nicht nur um akademisches Wissen, sondern vielfach um kaum dokumentiertes und auf vielfache Weise lokal geprägtes Routine- und Erfahrungswissen. Es hat seinen Grund, dass Investoren und Hedge-Fonds auf der Suche nach Spitzenrenditen nicht auf die Idee kommen, eine Armada von Hochschul-Absolventen anzuheuern und mit technologischen Spitzenapparaturen auszustatten. Es ist eben auch ein spezielles Wissen darum erforderlich, welches Wissen wann wie und wozu einzusetzen ist. Erforderlich ist ein komplexes Zusammenspiel heterogener Wissensarten, die untereinander und mit praktischen Routinen verflochten sind. ${ }^{3}$ Eine optimale Verbindung von Wissensformen und Handlungsroutinen, die sich in allen Kontexten und für alle Zwecke als überlegen erweisen würde, gibt es hier nicht. Es ist schwer, aus der best practice anderer zu lernen und riskant, den erfolgreichen eigenen Weg fort zu setzen, da genau dies in die Sackgasse führen kann. Es kann sein, dass zwei Unternehmen erfolgreich sind mit völlig unterschiedlichen Strategien. Und es kann das Aus für eine bestimmte Strategie bedeuten, wenn diese von allzu vielen Mitbewerbern kopiert wird und sich der Wettbewerbsvorteil in ein Negativ-Summen-Spiel verwandelt.

Wissen spielt eine entscheidende Rolle nicht nur bei der Ausführung von Tätigkeiten, sondern auch für ihre Koordination. Rationale Bürokratie ist idealtypisch an Fachwissen und Amtsautorität gebunden, also an eine bestimmte Verbindung von Wissen und Macht. Dabei wird - wiederum idealtypisch - davon ausgegangen, dass das relevante Wissen dort sitzt, wo die formelle Anordnungsbefugnis konzentriert ist. Im Extremfall muss der Vorgesetzte in der Lage sein, den Prozess, für den er verantwortlich ist, so weit zu überblicken, dass er seinen Mitarbeitern detaillierte Anweisungen auch zu Art und Ausgestaltung von Arbeitsund technischen Prozessen gegen kann. Hierfür benötigt er möglicherweise einen technischen Stab von Experten. Innerhalb des traditionellen Steuerungsregimes stellen diese Wissensarbeiter nur einen, wenn auch ständig wachsenden (und somit bald als Kostenproblem identifizierten) Teil der Belegschaft dar.

$\mathrm{Zu}$ einer veritablen Explosion der Anzahl der Wissensarbeiter kommt es indes paradoxerweise gerade dort, wo der Versuch gemacht wird, diese Expertenschicht traditioneller Wissensarbeiter zu entmachten und teilweise abzubauen. Partizipative Managementkonzepte und Formen dezentraler Selbststeuerung machen tendenziell jeden zum Wissensarbeiter. Wissen ist nicht länger Privileg des Managements; es wird tendenziell zur Aufgabe jedes Mitarbeiters, vor dem Hintergrund eines allen zur Verfügung stehenden Wissens über Kostenstrukturen, Markterfordernisse, Absatzentwicklung sowie interne und externe Mitbewerber die entsprechenden Konsequenzen zu ziehen (Kocyba 2003). Nichtwissen schützt nicht mehr vor den Folgen von (Nicht-)Entscheidungen.

Alle Formen der Buchführung und des Controlling setzen voraus, dass es möglich ist, auf eine zuverlässige Weise standardisiert Lagerbestände, Kundenaufträge, Geldbestände und so weiter zu erfassen und die dabei wechselseitige Abhängigkeit dieser Positionen zu überblicken. So lange Wissen als Herrschaftsprivileg galt, war es weder erforderlich noch erwünscht, dass das relevante Wissen in einer allgemein zugänglichen Weise verdichtet und dargestellt 
wurde. Gerade die Komplexität einerseits und die Vertraulichkeit der Daten und Analysen andererseits legitimierten die Machtbasis der Vorgesetzten. Deren Macht reichte letztlich so weit wie ihr Wissen und wurde durch dieses legitimiert. Die Unternehmenshierarchie erwies sich letztlich als Wissenshierarchie.

Dies bedeutete nicht, dass die oberen stets auch die Klügeren waren, aber sie waren es, die auf Informationen und Expertise zugreifen konnten und die im legitimen Besitz von Wissen waren, das im Munde anderer nur ein Gerücht oder Wissen vom Hörensagen gewesen wäre. Dieses Wissensszenario, in dem Wissen eine entscheidende Machtquelle war, führte zu bestimmten Formen der Auseinandersetzung. Der Kampf um die Kontrolle relevanter Ungewissheitszonen als eigentlicher Machtressource (vgl. Crozier, Friedberg 1993) machte es sinnvoll, Wissen nicht ohne weiteres preis zu geben. Gerade dieser Verdacht war es, der Taylors Anstrengungen beflügelte. Er war nicht der Meinung, dass Arbeiter dumm wären und daher die Unterstützung eines Experten benötigten, vielmehr sollten deren informelle Wissensund Kontrollspielräume schrittweise begrenzt und ausgeschaltet werden.

\section{Neubewertung von Erfahrungswissen}

Mittlerweile wird in der Managementliteratur ein anderes Arrangement favorisiert, demzufolge die Arbeiter ihr Wissen von sich aus im Rahmen von Verbesserungsprozessen offen legen (sollen). Dies wird mit Prämien honoriert. Sie sollen bei der Produktentwicklung einbezogen werden, ihr Erfahrungswissen wird neu bewertet (Böhle 2004). Die Vorgesetztenhierarchie ist keine Wissenshierarchie mehr, man arbeitet mit komplexen mehrsträngigen Hierarchien, der Mitarbeiter kann kompetenter sein als sein Vorgesetzter (und im Einzelfall auch mehr verdienen). Dessen Aufgabe ist eher die der Koordination, er muss nicht alles besser können und muss nicht alles kontrollieren können. Dies wird tendenziell zur Aufgabe der Mitarbeiter, die auf eine Nullfehler-Politik eingeschworen sind und sich selbst und ihre Kollegen beständig im Auge haben sollen. Detaillierte Anweisungen entfallen, wenn die Mitarbeiter selbst aus den verfügbaren Daten entsprechende Konsequenzen ziehen sollen. Es werden spezielle Aufgaben so gestaltet, dass Selbstorganisation wirksam werden kann und muss. Das Ergebnis ist, dass Wissen nicht nur Macht bedeutet, sondern auf eine bestimmte Weise auch deren Gegenteil, eine bestimmte Art von Ohnmacht, wie sie aus einer neuen Art der Verantwortlichkeit erwächst, die häufig ohne eine entsprechende Erweiterung formaler Befugnisse auskommen muss.

Damit jedoch dieses steuerungsrelevante Wissen vom „shop floor" aus aufgerufen und auch ergänzt oder verändert werden kann, muss es in der Regel standardisiert und vereinfacht werden. Wissen muss dann beispielsweise über Touchscreens in der Werkhalle abrufbar sein, muss stark auf Visualisierung und den Einsatz bildhafter Darstellungsformen setzen, um auch von Nichtexperten gedeutet werden zu können. Die diskursiven Elemente von Wissenspraktiken treten tendenziell zurück. Informationen können häufig unter Zugrundelegung eines vordefinierten Formats dezentral eingegeben werden oder können, soweit es sich beispielsweise um Maschinenlaufzeiten, Wartungsdaten oder bestimmte Anlageneinstellungen handelt, ohnehin automatisch erfasst werden.

Zumindest der Programmatik nach stehen auf jeder Hierarchiestufe dieselben Informationen zur Verfügung, auch die Chefs arbeiten lean und wollen sich nicht durch schiere Datenmassen entscheidungsunfähig machen lassen. Dieses Bild verdeckt allerdings, dass Wissen ja jeweils in einem bestimmten Interpretationskontext und Handlungshorizont sprechend wird. Was für den einen Leser der Daten feste Gegebenheiten oder Rahmendaten sind, stellt für den anderen ein zu gestaltendes Aufgabenfeld dar. Dieselbe Kennziffer sagt nicht allen dasselbe. Nach wie vor sind die Chancen recht ungleich verteilt, zusätzliche Informationen abrufen zu können, Zahlen in einen breiteren Vergleichskontext zu rücken und als Aussage in unterschiedliche Erzählungen zu integrieren. Einerseits sind jetzt alle Mitarbeiter legitime Träger wertvollen Wissens. Sie sind nicht länger Träger eines subversiven Gegenwissens, vielmehr ist ihr Praxiswissen jetzt offiziell als Ressource anerkannt, als Quelle potenzieller Verbesserungsvorschläge und innovativer Ideen. Dennoch hat diese neue Egalität erkennbare Grenzen.

Die Fähigkeit, Wissen wieder zu ,verflüssigen', unter Rekurs auf ein Wissen um seinen 
Fabrikations- und Funktionskontext flexibel zu handhaben, ist unterschiedlich verteilt. Der Aufforderungscharakter, den bestimmte Kennziffern besitzen, variiert nach Adressatengruppe. Erschwert ist in jedem Falle die Berufung auf Nicht-Wissen, und dies gerade in einer Welt, in der das Problem des Nicht-Wissens politisch bedeutsam wird. Wissen hat Grenzen der Haltbarkeit, der Gewissheit, der Genauigkeit und so weiter. Je mehr wir wissen, umso mehr wissen wir im Prinzip auch um die Grenzen unseres Wissens. Dies scheint aber dort nicht zu gelten, wo eine praktische Erfahrung mit der Fabrikation von Wissen, wie also bestimmte Messwerte oder Statistiken zu Stande kommen, fehlt oder nur eingeschränkt möglich wird. Wo die Fabrikation von Wissen (Knorr-Cetina 1984) nur in idealisierter Gestalt beobachtet werden kann, bildet sich leichter eine autoritär geprägte Haltung des Respekts vor einem vermeintlich unangreifbaren Wissen heraus. Dies veranlasst dazu, dem auf anonyme Weise zustande gekommenen Produkt dezentraler Wissensprozesse eine überpersönliche Gültigkeit zu attestieren. Suggestiv wirkt sich hier sicherlich noch die Tendenz zur visuell-grafischen Präsentation aus. Sie beherrscht inzwischen nicht nur die unternehmensinterne Kommunikation, sondern auch die Weise, in der Unternehmen mit der Öffentlichkeit oder ihren Aktionären kommunizieren.

Hier bricht sich nicht selten ein von suggestiven Bildern beherrschter ,Neo-Analphabetismus" Bahn. Die Frage, die sich hier stellt, ist die, ob die Entscheider dies als Inszenierung gegenüber einer gleichsam illiteraten Umgebung nutzen, während sie in Wirklichkeit auf der Basis sehr viel subtilerer Modelle entscheiden, oder ob diese anschaulich bebilderte ComicWelt zugleich ihren eigenen Entscheidungshorizont definiert. Strategische Bedeutung jedenfalls gewinnen die Experten, die für das kognitive und kommunikative Design dieser Welten verantwortlich sind. Das Ergebnis ihrer Tätigkeit wird nicht selten als neutrales Instrument akzeptiert und somit zur Prämisse weiterer Entscheidungen. Das Wissen, über das wir in Bezug auf unser eigenes Tun verfügen, ist also bereits durch eine Reihe von Standardisierungsprozeduren hindurchgegangen. Die eigene Erfahrung ist nur in beschränktem Maße unsere eigene. Dies gilt auch dort, wo Manager oder Eigentümer entscheiden (müssen). Auch dort ist es zuneh- mend weniger möglich, einfach ,aus dem Bauch heraus" zu entscheiden. In dem Maße, indem Accounting-Standards international angeglichen werden und in dem die Regeln der Kreditvergabe durch Basel $\mathrm{II}^{4}$ standardisiert werden (Pelzer 2007), ist zu erwarten, dass auch hier über die Kapitalkosten der Schatten standardisierter Wissenskulturen spürbar wird.

\section{Anmerkungen}

1) Als beispielhafte Ausnahme ist hier Foray (2004) zu nennen.

2) Zum Konzept der Rekontextualisierung siehe Schumm, Kocyba 1997.

3) Zur Diskussion über neue Formen der Wissensproduktion siehe Bender 2001.

4) Basel II meint die vom international zusammengesetzten Basler Ausschuss für Bankenaufsicht vorgeschlagenen Eigenkapitalvorschriften für Banken.

\section{Literatur}

Bender, G. (Hg.), 2001: Neue Formen der Wissenserzeugung. Frankfurt a. M.: Campus

Böhle, F., 2004: Die Bewältigung des Unplanbaren als neue Herausforderung in der Arbeitswelt - Die Unplanbarkeit betrieblicher Prozesse und erfahrungsgeleitetes Arbeiten. In: Böhle, F.; Pfeiffer, S.; Sevsay-Tegethoff, N. (Hg.): Die Bewältigung des Unplanbaren - Fachübergreifendes erfahrungsgeleitetes Arbeiten und Lernen. Wiesbaden: VS - Verlag für Sozialwissenschaften, S. 12-54

Crozier, M.; Friedberg, E., 1993: Die Zwänge kollektiven Handelns. Über macht und Organisation. Frankfurt a. M.: Campus

Drucker, P.F., 1993: Post-Capitalist Society. New York: Harper Collins Publishers

Foray, D., 2004: Economics of Knowledge. Cambridge (Mass.): MIT Press

Hack, L., 1998: Technologietransfer und Wissenstransformation. Münster: Westfälisches Dampfboot

Hayek, F.A., 1976: Individualismus und wirtschaftliche Ordnung. 2. Aufl. Salzburg: Wolfgang Neugebauer

Knorr-Cetina, K., 1984: Die Fabrikation von Erkenntnis. Frankfurt a. M.: Suhrkamp

Kocyba, H., 1999: Wissensbasierte Selbststeuerung. In: Konrad, W.; Schumm, W. (Hg.): Wissen und Arbeit. Münster: Westfälisches Dampfboot, S. $92-119$

Kocyba, H., 2003: Wissenspolitik im Unternehmen. In: Böschen, S.; Schulz-Schäffer, I. (Hg.): Wissen- 
schaft in der Wissensgesellschaft. Wiesbaden: Westdeutscher Verlag, S. 178-190

Kocyba, H.; Vormbusch, U., 2000: Partizipation als Managementstrategie. Frankfurt a. M.: Campus

Minssen, H., 1999: Von der Hierarchie zum Diskurs? Die Zumutungen der Selbstregulation. München: Rainer Hampp Verlag

Nonaka, I.; Takeuchi, H., 1997: Die Organisation des Wissens. Frankfurt a. M.: Campus

Ohno, T., 1993: Das Toyota-Produktionssystem. Frankfurt a. M.: Campus

Pelzer, P., 2007: Basel II, oder: die Einsicht in die unzureichende Verlässlichkeit reiner Quantifizierung. In: Mennicken, A.; Vollmer, H. (Hg.): Zahlenwerk. Kalkulation, Organisation und Gesellschaft. Wiesbaden: VS - Verlag für Sozialwissenschaften, S. 105-121

Schumm, W.; Kocyba, H., 1997: Re-Contextualization and Opportunities for Participation. In: Clausen, C.; Williams, R. (Hg.): The Social Shaping of Computer-Aided Production Management and of Computer-Integrated Manufacturing, Luxembourg: European Community, S. 49-62

Stehr, N., 2003: Das Produktivitätsparadox. In: Böschen, S.; Schulz-Schäffer, I. (Hg.): Wissenschaft in der Wissensgesellschaft. Wiesbaden: Westdeutscher Verlag, S. 77-93

Sveiby, K.E., 1998: Wissenskapital - das unentdeckte Vermögen. Landsberg: Verlag Moderne Industrie

Vormbusch, U., 2002: Diskussion und Disziplin. Gruppenarbeit als kommunikative und kalkulative Praxis. Frankfurt a. M.: Campus

\section{Kontakt}

Dr. Hermann Kocyba

Institut für Sozialforschung

Senckenberganlage 26, 60325 Frankfurt a. M.

Tel.: +49 (0) 69 / 756183 - 34

Fax: +49 (0) 69 / 749907

E-Mail: kocyba@em.uni-frankfurt.de

$\langle\gg$

\section{The Collaborative Work Concept and the Information Systems Support Perspectives for and from Manufacturing Industry ${ }^{1}$}

\author{
by António Brandão Moniz, Universidade Nova \\ de Lisboa
}

Most of the discussion and controversy on organisation of work concepts has been referenced to the manufacturing industry along the $20^{\text {th }}$ century: it started with the concept of "scientific management" from Taylor, and continued with the new ideas on the importance of human factors as Mayo pointed out in the $1930 \mathrm{~s}$. Immediately after the $2^{\text {nd }}$ World War Friedmann studied the human problems related to new manufacturing technologies and automation. And the late 1950 and 1960s were decades of strong debate on the socio-technics with the research at Tavistock Institute of London and the emergence of national programmes on new forms of work organisation. At the end of the last century the concept of collaborative work was developed together with the definition(s) of information systems and organisational design. However, the interest came from other production activities, like the services. This article analyses the approaches developed on these debates on the collaborative work and information system and its application to the manufacturing industry.

\section{Introduction}

There are newly emerging disciplines such as Computer Supported Cooperative Work that integrates the concepts of "collaborative work" and "information systems". In principle, such conceptual relation and the relevant methods deal with the development of information systems to support the strategy towards implementation of cooperative work. This means information sharing, qualifications and competences development, more democratic power relations, higher quality content of jobs, and so forth. However, this article tries to draw the attention to the fact that manufacturing is usually taken as an "old-fashioned" case to discuss the organisational change processes that imply participative 